\title{
Novel Strategies for Toxicological Risk Assessments
}

\author{
Adip Roy ${ }^{1}$ and Mohammad A. Akbarsha ${ }^{2}$ \\ ${ }^{1}$ Safety and Environmental Assurance Center, Unilever R\&D, Bangalore, India; ${ }^{2}$ Mahatma Gandhi-Doerenkamp Center (MGDC) \\ for Alternatives to the Use of Animals in Life Science Education, Bharathidasan University, Tiruchirappalli, India
}

http://dx.doi.org/10.14573/altex.1311081

\section{Introduction}

The Society of Toxicology (STOX), India, and Safety and Environmental Assurance Centre (SEAC), Unilever, organized a one day symposium on "Novel Strategies for Toxicological Risk Assessments" on September 4, 2013 at Unilever R\&D Centre, Bangalore, India. As animal testing for safety assessment of cosmetic ingredients and products is facing a likely ban in India, there is an increasing need to develop toxicological risk assessment strategies using non-animal methodologies, implying the need for a fundamental change in the way safety assessments are carried out. This symposium has been unique from the perspective of India in the sense that in spite of the rapid pace at which regulatory guidelines have been coming up in this country, the academic, scientific, industrial, and regulatory communities hardly have had occasion to meet to discuss non-animal methods in their respective endeavors. Given the increasing need for development of novel alternative methods and strategies for assessing toxicological safety, this symposium was organized to enhance awareness of newer toxicological and risk assessment approaches.

The symposium provided a platform for academic, industrial, and regulatory scientists and students to network and debate on current non-animal approaches available for toxicological risk assessment, to discuss the issues facing researchers in developing such methodologies, and to explore the need to develop capability and programs to address the issue.

\section{Technical sessions}

Dr Vilas Sinkar (Vice President and Site Leader Unilever R\&D, Bangalore) introduced the objectives of the symposium. Over many years toxicologists have relied on animal experiments to assess various toxicological endpoints and therefore assuring consumer safety without the generation of animal data is a considerable challenge. Although a lot of effort is needed to develop alternative methods for complex endpoints like systemic toxicity and reproductive toxicity, advances in modern biology, like systems biology and progress in genomics and bioinformatics, can help to reach these goals. Unilever, SEAC has played a significant role in the area of non-animal approaches to safety by partnering with academia and Dr Sinkar expressed his hope that the symposium would stimulate scientific discussions and identify a way forward in the area of animal alternatives for assessing toxicity.

Dr Carl Westmoreland (S\&T Director, SEAC, Unilever) talked about "Successes to Date with Non-Animal Approaches to Safety and Future Challenges". Talking about Unilever's conceptual framework for safety assessment being risk-based rather than hazard-based, Dr Westmoreland explained that Unilever's commitment to eliminating animal testing is underpinned by its work since the 1980s in developing and using alternatives to animal tests for assessing safety. The talk covered the progress in the development, validation, and acceptance of non-animal approaches to toxicological risk assessments as well as developments in exposure science that can help reduce the numbers of animals used for safety assessment. Toxicology and ecotoxicology that underpins consumer and environmental risk assessment is rapidly evolving. Effort is being made in the context of the OECD "Adverse Outcome Pathway" (AOP) work program, where Unilever and their scientific partners are working to put in place the tools and novel thinking needed to implement "Toxicity Testing in the $21^{\text {st }}$ Century" (TT21C)/ AOP-based consumer and environmental risk assessments. In so doing, the aim is to ultimately remove dependence on apical endpoint studies and bring novel science to the decisions made on the safe use of chemicals within consumer products, he said.

Benedict Mascarenhas (Chairman \& Managing Director, EnvisBE Solutions Pvt. Ltd) spoke on the "Regulatory Requirements for Cosmetics in India Related to Toxicity Testing". The cosmetics industry has seen significant developments in the recent past with the development of novel ingredients and with multi-benefit products containing multiple active substances. Safety testing therefore remains an important aspect of cosmetic product development. One of the recent international developments associated with an attempt towards responsible cosmetic product development is the banning of animal testing, spearheaded by the EU and also recently adopted by India. This poses both a challenge and an opportunity. The challenge is primarily the requirement for rapidly establishing alternative, validated methods for all areas of cosmetic toxicity assessment. The opportunity for India is to lead/participate in the validation programs in areas of strategic importance. $\mathrm{He}$ noted that adopting integrated testing strategies (ITS) involv- 
ing a combination of validated alternative methods (VAM) along with the weight of evidence (WoE) approach is the need of the hour.

Dr Nilanjan Guha (Agilent Technologies India Pvt. Ltd.) spoke on "Systems Toxicology: The Need of the $21^{\text {st }}$ Century". He emphasized that toxicology researchers are embarking on multi-omics approaches to refine existing methods to better assess safety issues related to a wide range of substances developed by the pharmaceutical, chemical, agri-bio, and cosmetic industries. Omics technologies help to understand complex biological systems by finding meaningful signals amid the background noise of cellular processes. Not only will these tests allow high-throughput screening of compounds for toxic effects; they also are expected to provide more accurate assessments of toxicity in humans than current animal-based approaches. He further presented results from a proof-of-concept study demonstrating the utility of applying an integrative approach to obtain a complete cellular response profile by coanalyzing data acquired from complementary technologies.

Kas Subramanian (Chief Scientific Officer, Strand Life Sciences) talked about "Assessing hepato-toxicological risk by quantitative modeling of liver metabolic pathways". Several methods have been developed to predict drug-induced liver injury with varying degrees of accuracy and applicability. Strand Life Sciences has taken a systems approach to address this problem by mapping the pathways that underlie the major forms of liver injury observed in the clinic. A mathematical model of the liver has been developed based on the kinetics of the enzymes involved in the essential pathways. This model accurately represents energy and anti-oxidant metabolism in that it is able to reproduce liver homeostasis. It can model liver injury upon simulation of energy or anti-oxidant depleting stresses. It also replicates liver and whole system behavior on induction of stresses that restrict bile flow. The model evolves to demonstrate fatty liver as a response to mitochondrial dysfunction as well as disturbances in fatty acid uptake. Thus the model uses insights gained from the modeling to design in vitro assays that measure key perturbations and use the in vitro-in silico combination to assess and predict hepatotoxic risk, he said.

Dr K. S. Rao (Senior Research Director and Head of Safety Assessment, Syngene International Ltd.), presenting on "Risk Assessment of Nanomaterials with Emphasis on using Alternatives to Animal Testing" expressed that widespread application of nanomaterials confers enormous potential for human exposure and environmental release. The properties of nanoparticles present new challenges to understanding, predicting, and managing potential adverse health effects following exposure. The risk assessment paradigm (hazard and exposure assessment, dose response, and risk characterization) used for conventional substances will also apply to nanomaterials. However, the hazard assessment of nanoparticles does pose formidable challenges owing to the uncertain and unpredictable nature/state of the nanoparticles in diverse media. None of the validated alternative methods currently available for con- ventional chemical substances has been validated specifically for nanomaterials. He said that this poses an obstacle to safety assessment of cosmetic nanomaterials, and further research work is needed in this area.

Dr Sudin Bhattacharya (Senior Research Investigator, Institute for Chemical Safety Sciences, The Hamner Institutes for Health Sciences) presented his work on "Mapping nuclear receptor-mediated toxicity pathways in the liver". Nuclear receptor activation in the liver leads to coordinated alteration of the expression of multiple gene products with attendant phenotypic changes. In studies on the signaling network induced by the peroxisome proliferator-associated receptor $\alpha$ (PPAR $\alpha)$ in primary human hepatocytes, his group has assessed all differentially expressed genes over all treatments. Using novel bioinformatics methods and available databases several key transcription factors involved with regulation of the non-genomic targets can be identified. He explained that these novel strategies for mapping transcriptional networks are evolving and are necessary in moving from toxicogenomic methods that derive signatures of activity to methods that establish pathway structure, showing the coordination of the activated nuclear receptor with other signaling pathways.

Anup Mammen Oommen (Head, Network Biology Group, Connexios Life Sciences PVT Ltd.) in his presentation on "Assessing toxicity in cells: A network biology approach" explained that Connexios Life Sciences has developed a rigorous pathology centered network biology approach to discover small molecule based therapies for type 2 diabetes and other chronic diseases. The network biology approach entails mapping the complex cellular pathology of disease to molecular networks that would be the basis for developing tools to aid drug discovery and development. Connexios cell specific network models consist of cellular processes interconnected in a causal relationship that has the potential to explain development of the cell specific toxicity. Intensive analysis of these networks using various toxicology related omics data enables identifying the molecular fingerprints of xenobiotic induced toxicity and resultant cellular adaptations to toxic insults, which constitute "effective molecular networks". Extensive in vitro validation of these effective molecular networks has enabled developing predictive toxicity marker panels with which in vitro findings can be translated to the clinic.

Dr A. B. Pant (Sr. Scientist \& In-Charge, In Vitro Toxicology, Indian Institute of Toxicology Research) presented his work on "Application of human cord blood stem cell derived neuronal cells in pathway specific developmental neurotoxicity". He explained that developmental neurotoxicity assessment is critical for environmental chemicals, drugs, and new chemical entities and stated that mechanistic studies have been hampered primarily due to non-availability of developing brain tissues. Stem cell technology provides a new tool for better understanding the toxicity mechanisms involved and how to potentially predict and avoid toxicity in humans. They have utilized the development and pluripotency potential of human umbilical cord blood stem cells to convert them into neural subtypes and 
subsequently as the tool to assess developmental neurotoxicity. Responsiveness of differentiating cells was studied following the exposure to known neurotoxicants, e.g., the organophosphate pesticide monocrotophos, to identify the stage and pathway specific biomarkers of neural development, injury, and repair. Studies also are being carried out to identify cellular and molecular targets for neuroprotection in cells exposed to toxic insult and the effects of known neuroprotectants.

Dr Paul Carmichael (SEAC, Unilever R\&D), presenting on "Toxicity Testing in the $21^{\text {st }}$ Century: Taking a Pathway-led Approach" highlighted the US National Research Council's publication "Toxicity Testing in the $21^{\text {st }}$ Century: A Vision and a Strategy" and outlined an approach to safety assessment that "could transform toxicity testing from a system based on whole-animal testing to one founded primarily on in vitro methods that evaluate changes in biologic processes using cells, cell lines, or cellular components, preferably of human origin". His talk emphasized that understanding of key biological pathways and adverse outcome pathways can help develop the strategy and vision from the conceptual to the practical, developing the necessary steps to use this vision as the basis of the safety assessment of new ingredients in consumer products without animal testing. Combining the outputs of in vitro assays and in silico networks can help to make a decision on the in vivo human safety estimation.

Additionally, there were poster presentations on "Systems Biology for Toxicity Testing - Emphasis on Synergism of Top-Down and Bottom-Up Approaches: Examples from $C y$ tochrome P450 Enzyme System and Beyond' by Bagavathy S. Karthikeyan, Subbaiah Parthasarathy, and Mohammad A. Akbarsha from Bharathidasan University, Tiruchirappalli, and "Detection of Oxidation/Alkylation DNA damage in the comet assay using lesion-specific repair enzymes" by Mahesh Batakurki and T. K. Sivaram from Unilever R\&D, Bangalore.

\section{Discussion and conclusions}

The symposium culminated in a lively panel discussion, chaired by Dr K. C. Gupta (Director, Indian Institute of Toxicology Research (IITR), Lucknow). Topics including the cur- rent status of research on alternatives (current projects, gaps, funding), education and training in alternatives, policy and regulations were addressed. Some of the outcomes were:

1) Learn from the experience from many years of research that has been carried out in the EU and USA on animal alternatives: The EU initiated action to ban animal use in cosmetic testing in 1993 and within a span of 10 years research groups developed viable non-animal methodologies like 3D culture and reconstructed tissue models. These models have been validated and recognized by ECVAM, ICCVAM, and OECD. Now that the guidelines for cosmetics testing in India are being harmonized with those in the EU, the scientific community in India, including in the industry, should develop viable alternative modalities appropriate for the Indian context, and not rely entirely on the models developed in the EU and US.

2) We should have a vision on how we will build on this symposium - we need to develop a roadmap: The change should start from the academia. Training and courses on alternative methodologies, as is offered at Mahatma Gandhi-Doerenkamp Center for Alternatives, Bharathidasan University, should be organized, for which the regulatory authorities should extend fiscal and technical support. The academia should build on these programs to produce a viable roadmap for the Indian alternatives scenario. There is a need to build capability and upgrade skills, especially in areas of modeling. The Society of Toxicology (STOX) will help in all the endeavors connecting alternatives and toxicity testing.

3) Academia-industry and industry-industry partnerships are critical in addressing the issue of alternative methods. Industry should participate in developing models and methodologies through collaboration.

It was concluded that a significant opportunity exists for Indian scientists to contribute novel science and thinking as part of the global research effort to find novel ways of assuring safety without animal testing. It is hoped that this symposium will stimulate further scientific discussions towards mapping a way forward in the area of animal alternatives for assessing toxicity. 